Gut, 1974, 15, 801-804

\title{
The relationship and epidemiology of acute terminal ileitis and Crohn's disease
}

\author{
J. KEWENTER, L. HULTÉN, AND N. G. KOCK \\ From the Departments of Surgery II and III, University of Gothenburg, Gothenburg, Sweden
}

SUMmARY The frequency of admission for acute terminal ileitis, Crohn's disease, and acute mesenteric lymphadenitis has been compared from the years 1951 to 1970 , and the long-term results of operation for acute terminal ileitis in 1951-1965 have been examined.

Whereas the admission rates for both acute terminal ileitis and Crohn's disease rose during the review period, that for acute lymphadenitis tended to fall slightly. Sixty-eight of 72 patients operated upon by appendicectomy who had acute terminal ileitis were followed up. One of these, with small intestinal stenosis at the initial operation, proved to have histological evidence of Crohn's disease, and a further nine have since developed unequivocal evidence of the disease. A cutaneous fistula developed in only one patient after appendicectomy. It is concluded that appendicectomy can safely be performed in acute terminal ileitis provided that the caecum is not inflamed and that Crohn's disease seldom develops as a sequel.

Opinions differ as to whether acute terminal ileitis is a separate disease or an acute form of the classical chronic granulomatous Crohn's disease. Thus, Crohn (1965) suggested that acute terminal ileitis is an acute form of the disease in the terminal ileum, an assumption that was not accepted by Gump, Lepore, and Barker (1967) nor by Thomasson and Havia (1973).

In order to gain information about the prognosis in patients with acute terminal ileitis, established at laparotomy, and to investigate the relationship between it and Crohn's disease, the records of all patients treated for clinically diagnosed acute terminal ileitis at Sahlgren's Hospital between 1951 and 1965 were investigated and the patients were followed up. Sahlgren's Hospital is the only hospital admitting acutely ill patients from Gothenburg (mean population 395000 during these years). Furthermore, the admission rate of patients with acute terminal ileitis during this period was compared with the frequency of Crohn's disease in the small and large bowel.

\section{Patients}

During the period 1951-1965, 10625 adult patients were admitted to Sahlgren's Hospital with the clinical diagnosis of appendicitis. All patients had been

Received for publication 27 June 1974. acutely ill with abdominal symptoms leading to an exploratory laparotomy for suspected appendicitis.

Of these patients, 925 were classified as having mesenteric lymphadenitis, ie, having a normal appendix and intestine but demonstrating enlarged mesenteric lymph nodes. In 72 cases, the operation revealed an inflamed, thickened, hyperaemic terminal ileum, often with enlarged lymph nodes in the ileo-caecal junction, ie, acute terminal ileitis.

The mean age was 31 years (range 17-69 years). The length of the follow up varied between five and 20 years with a mean observation time of 10 years.

A letter of inquiry was sent to all the patients who had been operated upon for acute terminal ileitis. We were unable to find four patients and, therefore, 68 patients wert left for the follow up, 33 of them men. All patients who complained of abdominal discomfort (21) were examined and a barium study of the small and large intestine as well as sigmoidoscopy and routine blood tests were performed.

The frequency of acute terminal ileitis during the four consecutive five-year periods between 1951 and 1970 was calculated and compared with the frequency of Crohn's disease, mesenteric lymphadenitis, and appendicectomies.

\section{Results}

THE OUTCOME FOR PATIENTS WITH ACUTE TERMINAL ILEITIS

Sixty-seven of the 68 patients had the appendix 801 
removed after admission and a right hemicolectomy was performed on one patient due to the risk of obstruction. The specimen from this patient showed Crohn's disease. Eight patients have been reoperated upon due to persistent or recurrent symptoms at varying times after the initial appendicectomy. Four of these patients were reoperated upon within one year with a right hemicolectomy and ileotransversostomy, one of these for a cutaneous fistula after the appendicectomy. The remaining four patients had a right hemicolectomy following appendicectomy after five, seven, eight, and 15 years, respectively, due to severe recurrence of the inflammatory bowel disease. All specimuns removed were investigated (Dr Christer Åhrén) postoperatively, and all showed microscopically the typical picture of Crohn's disease. The specimen from the patient with a cutaneous fistula showed that the caecum was also involved by the disease.

Of the remaining 59 patients, an additional 21 complained of abdominal pain or discomfort. All these 21 patients were examined for evidence of Crohn's disease in the small or large bowel by barium enema, a small bowel follow through, physical examination, and sigmoidoscopy. All were normal except one patient whose small bowel follow through showed the typical radiological picture of Crohn's disease in the terminal ileum. This patient was therefore diagnosed as having Crohn's disease, bringing the total number of patients with this disease to $10(14 \%)$ (table I).

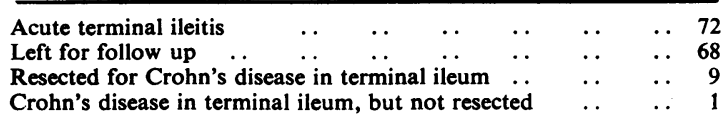

Table I Analysis of series

FREQUENCY OF ACUTE TERMINAL ILEITIS AND ACUTE MESENTERICLYMPHADENITIS COMPARED WITH THAT OF CROHN'S DISEASE

The admission rate during the four five-year periods between 1951 and 1970 of patients subjected to appendicectomy, as well as the frequency of patients with acute terminal ileitis, Crohn's disease only in the small bowel, the total number of patients with Crohn's disease and mesenteric lymphadenopathy are shown in table II.

The frequency of acute terminal ileitis showed a clear increase between the first two-year period investigated. A similar increase in frequency but appearing later was found for Crohn's disease of the small bowel only. This finding applies also to the frequency for the total number of patients with this disease. The frequency for all these three groups seems to be constant during the last decade (1961-1970).

The admission rate of patients subjected to appendicectomy as well as that of patients with mesenteric lymphadenitis, as established at laparotomy, showed a slight decrease during the last decade.

\section{Discussion}

Opinions differ as to whether acute terminal ileitis is a separate entity or an acute form of the classical chronic granulomatous Crohn's disease. Crohn (1965) stated that acute terminal ileitis was a true clinical and pathological entity which is the precursor of chronic granulomatous ileitis. This opinion was based on his collected experience from 42 patients who were followed up for approximately 15 years. In half of these patients, later surgery for chronic disease was required, but spontaneous 'cure' resulted in the remainder. In the present study, $15 \%$ of the patients with acute terminal ileitis were later found to have Crohn's disease. These cases constitute $11 \%$ of the total number of patients with Crohn's disease in the small intestine only and $3.7 \%$ of all cases of Crohn's disease admitted to the hospital during this period. Gump (1967) and Thomasson and Havia (1973), on the other hand, found no evidence at all for progressive disease in their conservatively treated series of patients with acute terminal ileitis. To our mind, however, there is no doubt that the classical chronic Crohn's disease can manifest itself in an acute form and thereby be very similar to acute terminal ileitis. At laparotomy, it is impossible to distinguish

\begin{tabular}{lllll}
\hline & $1951-1955$ & $1956-1960$ & $1961-1965$ & $1966-1970$ \\
\hline Appendicectomy & 192 & 181 & 169 & 127 \\
Mesenteric lymphadenitis & 16 & 17 & 13 & $9 \cdot 8$ \\
Acute terminal ileitis & 0.4 & 1.2 & 1.7 & 1.1 \\
Crohn s disease of the small intestine & 0.5 & 0.5 & 1.7 & $1 \cdot 7$ \\
Total number of cases of Crohn's disease & 1.2 & 1.6 & 6.3 & 6.3 \\
\hline
\end{tabular}

Table II Incidence of appendicectomies, mesenteric lymphadenitis, acute terminal ileitis, Crohn's disease of the small intestine and the total frequency of Crohn's disease per 100000 inhabitants in Gothenburg during four five-year periods between 1951 and 1970 (358-451 000 inhabitants) 
between the patients in whom the disease will become chronic and those in whom it will heal without further complications.

In addition to Crohn's disease, different aetiologies have been attributed to the clinical and macroscopical manifestations of the disease known as acute terminal ileitis. Thus, it has been reported from Japan (Hayasaka, Ishikura, and Takayama, 1971) that the fish nematode Anisalis may cause this disease, and reports from Europe and the United States (Winblad, Niléhn, and Sternby, 1966; Weber, Finlayson, and Mark, 1970) have implied that the species Yersinia can induce acute terminal ileitis but it has never been shown that ileitis caused by Anisalis or Yersinia progresses to Crohn's disease in the terminal ileum. In view of the still disputed cause of acute terminal ileitis and the variation in the incidence of Crohn's disease in different countries, it is hardly surprising that the number of patients with acute terminal ileitis who have developed Crohn's disease varies considerably in different reports.

As serological and bacteriological methods are developed, it may be possible to separate certain aetiological groups from the idiopathic group now known as acute terminal ileitis and to classify these subgroups according to aetiology. So far, the follow up of patients with acute terminal ileitis is the only way of identifying those patients in whom the acute attack was due to Crohn's disease. The results of the present investigation also indicate that, from a practical point of view, it is impossible at laparotomy to distinguish between those patients in whom the disease will become progressive and those in whom it will resolve spontaneously without further complications. Since the conservative or expectant attitude will allow for a reasonably low risk of misinterpretation, there are no indications for resection or bypass operation at the time of the acute laparotomy unless a specific complication is noted.

In the past it was proposed that appendicectomy should be avoided in these patients due to the high risk of postoperative fistula. Only one of the patients in this study developed this complication. A right hemicolectomy was later performed in this case and histology showed Crohn's disease in the terminal ileum and caecum. A low frequency of postoperative fistula formation in acute terminal ileitis was noted also by Atwell, Duthie, and Goligher (1965), indicating that appendicectomy can be performed at the primary procedure with negligible risk of fistula formation, unless the appendix base is involved in the inflammatory reaction. There is a strong justification for appendicectomy, however, because appendicitis can be excluded from the differential diagnosis in any future attack.
In the patients who developed Crohn's disease, nine out of 10 had symptoms from the bowel disease that made a resection necessary.

The purpose of the present investigation was also to collect information regarding the relative frequency of acute terminal ileitis versus that of Crohn's disease and acute mesenteric lymphadenitis during the corresponding time periods. Crohn's disease of the small intestine showed a significant increase between 1951-1960 and 1961-1965 as did the total number of patients with Crohn's disease. During the last decade, no additional increase could be observed. An increased incidence of Crohn's disease has previously been reported from Sweden, but the present results are surprisingly highcompared with previously reported figures (Krause, Bergman, and Norlén, 1971).

The frequency of acute terminal ileitis increased in a similar fashion also during 1956-1960, but remained stable during the last three five-year periods. This trend is also in agreement with a recent report from Copenhagen (Höj, Brix Jensen, Bonnevie, and Riis, 1973). The frequency of acute mesenteric lymphadenitis has remained fairly stable during the whole period. If anything, a small decrease could be observed. The parallel increase in the frequency of Crohn's disease in the small intestine and that of acute terminal ileitis is interesting. It might indicate that the two conditions are related in one way or another. The stable, or possibly slightly decreased, frequency of mesenteric lymphadenopathy is surprising, particularly since it has been proposed that the disease is related aetiologically to acute terminal ileitis (Weber et al, 1970).

The nomenclature in this field is confusing, perhaps mainly because of the similarity in anatomical localization and macroscopic appearance of the classical Crohn's disease with that seen in patients with an acutely inflamed terminal ileum but this does not justify a general classification of these patients as suffering from 'acute' Crohn's disease. One must bear in mind the implications this can have for the patient with respect to insurance and the psychological problems of being classified, incorrectly, as suffering from a chronic disease. The concept 'acute Crohn's disease' is misleading. The follow up of the patients with acute terminal ileitis is the only way to identify those patients in whom the acute attack has been due to Crohn's disease.

\section{References}

Atwell, J. D., Duthie, H. L., and Goligher, J. C. (1965). The outcome of Crohn s disease. Brit. J. Surg., 52, 966-972.

Crohn, B. (1965). The pathology of acute regional ileitis. Amer. J. dig. Dis., 10, 565-572. 
Gump, F. E., Lepore, M., and Barker, H. G. (1967). A revised concept of acute regional enteritis. Ann. Surg., 166, 942-946.

Hayasaka, H., Ishikura, H., and Takayama, T. (1971). Acute regional ileitis due to Anisakis Larvae. Int. Surg., 55, 8-14.

Höj, L., Brix Jensen, P., Bonnevie, O., and Riis, P. (1973). An epidemiological study of regional enteritis and acute ileitis in Copenhagen county. Scand. J. Gastroent., 8, 381-384.

Krause, U., Bergman, L., and Norlén, B. J. (1971). Crohn's disease: a clinical study based on 186 patients. Scand. J. Gastroent.,
6, 97-108.

Thomasson, B., and Havia, T. (1973). Is acute terminal ileitis a precursor of Crohn s disease? Acta Chir. scand., 139, 192-193. Weber, J., Finlayson, N. B., and Mark, J. B. D. (1970). Mesenteric lymphadenitis and terminal ileitis due to Yersinia Pseudotuberculosis. New Engl. J. Med., 283, 172--174.

Winblad, S., Nilêhn, B., and Sternby, N. H. (1966). Yersinia enterocolitica (Pasteurella $X$ ) in Human enteric infections. Brit. med. J., 2, 1363-1366. 